\title{
Rhinitis as a risk factor for depressive mood in pre- adolescents: a new approach to this relationship
}

\author{
Palma Audino ${ }^{1}$, Stefania La Grutta ${ }^{1}$, Fabio Cibella ${ }^{1}$, Sabina La Grutta ${ }^{2}$, Mario R. Melis ${ }^{1}$, Salvatore \\ Bucchieri $^{1}$, Pietro Alfano ${ }^{1}$, Salvatore Marcantonio ${ }^{3}$ \& Giuseppina Cuttitta ${ }^{1}$ \\ ${ }^{1}$ National Research Council of Italy, Institute of Biomedicine and Molecular Immunology, Palermo, Italy; ${ }^{2}$ Department of Psychology, University \\ of Palermo, Palermo, Italy; ${ }^{3}$ Department of Statistical and Mathematical Sciences, University of Palermo, Palermo, Italy
}

To cite this article: Audino P, La Grutta S, Cibella F, La Grutta S, Melis MR, Bucchieri S, Alfano P, Marcantonio S, Cuttitta G. Rhinitis as a risk factor for depressive mood in pre-adolescents: a new approach to this relationship. Pediatr Allergy Immunol 2014: 25: 360-365.

\section{Keywords}

allergic sensitization; anxious mood; asthma; depressive mood; household crowding index; partial directed acyclic graph; preadolescents; rhinitis

\section{Correspondence \\ Giuseppina Cuttitta, MD, National Research Council of Italy, Institute of Biomedicine and Molecular Immunology "A. Monroy", Via Ugo La Malfa 153, 90146 Palermo, Italy. Tel.: +390916809137 \\ Fax: +390916809122 \\ E-mail: giuseppina.cuttitta@ibim.cnr.it}

Accepted for publication 11 February 2014

DOI:10.1111/pai.12215

\begin{abstract}
Background: Respiratory allergic symptoms impact on social life and school activities, influencing the patient's mood states. We evaluated the relationships between allergic respiratory diseases and depressive/anxious mood in a large sample of Italian middle school students, using the partial directed acyclic graph (P-DAG).

Methods: We studied 1283 subjects aged 10-13. A health respiratory questionnaire including questions relevant to socioeconomic status (HCI) and a test for depression and anxiety were administered. All subjects performed spirometry and skin prick tests.

Results: A causal role of rhinitis on depression was found: the likelihood of being depressed increased from 11.2 to $17.7 \%$, when rhinitis was present. Moreover, a direct effect of low HCI on depressive mood was shown $(p<0.0001)$ as well as the correlation between anxiety and depression $(\mathrm{p}<0.0001)$. Gender was not a direct causal factor for depressive mood, but their relation was mediated through anxious mood. Anxiety appeared to have a stronger association with depression than gender. Allergic sensitization was significantly related to both asthma and rhinitis $(\mathrm{p}<0.0001$, respectively). Asthma and rhinitis were also directly associated $(p<0.0001)$. Conversely, asthma was not directly associated with depressive mood, but their relation was mediated through rhinitis. Body mass index (BMI) and impaired lung function (IPF) were not associated with the other variables.

Conclusions: The use of this novel approach to analyzing the dynamic relationships allowed us to find a causal role of rhinitis on depressive state. Moreover, anxious condition and low socioeconomic status contributed to induce depressive mood.
\end{abstract}

Respiratory allergic symptoms have a considerable impact on social life, school activities, influencing the patient's mood states (1). There has been growing interest in the relationships between psychologic factors and allergic respiratory diseases, mostly in children with chronic diseases, such as atopic disorders (2), seeking a better explanation of the psychologic burden posed by respiratory allergic diseases on anxiety and depression symptoms (3).

Rhinitis and asthma are global health problems, increasingly in childhood (4). In Italy, a $35 \%$ prevalence of rhinitis symptoms in the last 12 months and a prevalence of $4.5 \%$ of current asthma among 13-14 yr of age subjects were reported in a cross-sectional survey of 2002 (5). Atopic status is one of the main determinants of rhinitis (6), whereas many factors, other than atopic status, play a role in bronchial asthma.

Previous data regarding the impact of childhood allergic asthma have reported a strong negative effect on wellness when associated with rhinitis, producing detrimental effects on physical, psychologic, and social dimensions of life (7).

However, evaluations of anxiety and depressive states aimed at emphasizing the coexistence of psychologic conditions, and atopic diseases are not often included in epidemiological surveys (2).

Currently, the association between allergic rhinitis and psychologic characteristics in pediatric age remains poorly explored. 
This study evaluates (i) the relationships between respiratory symptoms (such as asthma, rhinitis) and depressive/anxious mood, (ii) any effects of confounding and/or mediating factors in these relationships by the partial directed acyclic graph (P-DAG).

\section{Methods}

This cross-sectional study was conducted on a sample of middle school students, aged 10-13, in Palermo, Italy. In this multistage cluster sample, first-stage units were schools, and second-stage units were students. After subdividing the city in three geographic zones (coastal, downtown, and hilly), 16 Palermo schools were selected as representative of the city, whereas participants were autoselected. Initial sample size was 2150 students, of which 1336 decided to participate $(62 \%$ response rate); fifty-three were deleted because of null values, leaving 1283 to be analyzed. To obtain data on age, gender, presence of asthma and/or rhinitis, and home characteristics, each subject completed a standardized questionnaire based on the SIDRIA survey $(5,8)$. Household crowding index (HCI), considered a proxy for socioeconomic status (9), was defined as the number of coresidents per household, divided by the number of rooms, excluding the kitchen and bathrooms: it was dichotomized as $\leq 1$ or $>1$.

All subjects performed spirometry and skin prick tests and completed psychologic assessment testing for depression and anxiety characteristics using the Depression and Anxiety in Youth Scale (TAD) (10).

The study was approved by the Institutional Ethics Committee. All parents gave written informed consent. The individual privacy of clinical data was guaranteed under Italian law.

\section{Respiratory health questionnaire}

A personal history of asthma was defined as a positive answer to the question 'Have you ever had asthma', plus at least one wheezing episode in the previous 12 months (current asthma). Rhinitis was defined as a positive answer to the question: 'Have you ever had a problem with sneezing, or runny, or blocked nose apart from common cold or flu in the past 12 months' (11). The analysis considered: subjects with asthma, subjects with rhinitis, subjects with both asthma and rhinitis, and control subjects with neither asthma nor rhinitis.

\section{Skin prick test}

All subjects were submitted to skin prick tests to common aeroallergens, according to EAACI recommendations (12). Allergic sensitization was defined as a positive skin prick to at least one allergen.

\section{Spirometry}

Height and weight were measured in all the children in standing position without shoes, and BMI was computed as weight $/$ height ${ }^{2}\left(\mathrm{~kg} / \mathrm{m}^{2}\right)$. Pulmonary function tests were performed using a portable spirometer (MicroLoop, Micro
Medical, Chatham Maritime, Kent, UK). Forced expiratory volume in one-second $\left(\mathrm{FEV}_{1}\right)$, forced vital capacity $(\mathrm{FVC})$, and maximum midexpiratory flow $\left(\mathrm{FEF}_{25-75 \%}\right)$ were measured according to ATS/ERS guidelines (13), and the best FVC and $\mathrm{FEV}_{1}$ were retained. Spirometric predicted values were those from Pistelli et al (14). IPF was defined as $\mathrm{FEV}_{1} / \mathrm{FVC}$ percentage below the 5 th percentile of normal distribution (below $87 \%$ of predicted for boys and below $90 \%$ for girls). Overweight-obese children were defined as having a BMI $\geq 25 \mathrm{~kg} / \mathrm{m}^{2}$ (15).

\section{Psychologic assessment}

Depressive and anxious mood were assessed by the TAD (10), a measure of depression and anxiety in children and adolescents. This questionnaire provides three sources of data regarding thoughts, emotions, and behaviors. We used only the children scale self-assessment. It contains 11 items for depression and 11 for anxiety; subjects select a 4-point Likert scale rating, from 1 (not at all) to 4 (almost all the time). Standard scores, with mean of 100 and standard deviation of 15 , were assigned to the scale. A score higher than 115 was considered indicate the presence of clinical depressive and anxious mood.

\section{Data analysis}

To depict qualitative relationships among variables, a partial directed acyclic graph (P-DAG) (16) was created. A P-DAG evaluates the probabilistic relationships among all the variables in the network, where relationships are represented by direct arrows and variables are represented by nodes. This is a particularly useful tool to show how the probability of one variable is going to change due to the observation of other variables, regardless of sample size. Given observational data, the P-DAG is a powerful tool to inspect associational (segments) and causal (arrows) relationships among variables (17). The probabilistic relationships are defined through two components: a set of nodes and a set of conditional probability tables. The set of all variables that makes the node independent from the rest of the network represents the Markov blanket of such node. This means that the Markov blanket of a node is the only knowledge needed to predict the role of variable in the network.

The network was built using the 'grow-shrink' algorithm (18) through parametric chi-square tests. Conditional probability tables were used to describe quantitative association, and relative difference was used to evaluate the strength of effects (see Appendix S1).

One-way analysis of variance (ANOvA) was performed by chisquare tests. A probability level of $\mathrm{p}<0.05$ was selected as statistically significant. All computations were performed by bnlearn package of $\mathrm{R}$ suite (19).

\section{Results}

Anthropometric and clinical characteristics of the original sample (No. 2150) and the studied sample (No. 1283) are 
shown in Table 1. No significant differences among the analyzed variables were found.

The prevalence of general and clinical characteristics in the studied sample separately for gender is shown in Table 2. The prevalence of allergic sensitizations was higher in males than in females, whereas the prevalence of anxious mood was higher in females than in males. No significant differences were found for all the remaining variable, separately for gender.

The prevalence of anxiety was $20 \%$ among rhinitis and $15.8 \%$ among asthmatics, whereas the prevalence of depressive symptoms was $17.6 \%$ among rhinitis and $10.5 \%$ among asthmatics.

The P-DAG (Fig. 1) summarizes the possible relationships among variables, considering what is known about this field. Gender was a direct causal factor of allergic sensitization $\left(\chi^{2}\right.$, $\mathrm{p}<0.0001$ ), which in turn mediated its relationships with both asthma and rhinitis $\left(\chi^{2}, \mathrm{p}<0.0001\right)$. Asthma and rhinitis were also directly associated $\left(\chi^{2}, \mathrm{p}<0.0001\right)$, as were anxious and depressive $\operatorname{mood}\left(\chi^{2}, \mathrm{p}<0.0001\right)$. Asthma was not directly associated with depressive mood, but their relation was mediated through rhinitis. Similarly, gender was not a direct causal factor for depressive mood, but their link was mediated through anxious mood, which was directly influenced by gender. Furthermore, allergic sensitization was not a direct causal factor for anxious mood, but their relationship was confounded by gender $\left(\chi^{2}, p<0.001\right)$. Among the other variables analyzed, a direct link between $\mathrm{HCI}$ and depressive mood was also shown $\left(\chi^{2}, \mathrm{p}<0.0001\right)$; conversely, BMI and IPF were not associated with the other variables.

We studied the distribution of each combination of rhinitis and asthma for subjects with and without allergic sensitization. When allergic sensitization was present, the likelihood to being both asthmatic and rhinitic increased more than threefold $(1.9 \% \rightarrow 7.1 \%)$. The effect of allergic sensitization on rhinitis was weaker; in fact, when allergic sensitization was present, the likelihood of being rhinitic increased about one-fourth

Table 1 General characteristics of the sample studied and the original sample

\begin{tabular}{lcc}
\hline & Studied sample & Original sample \\
& $\mathrm{N}=1283$ & $\mathrm{~N}=2150$ \\
\hline Gender (M/F) & $645 / 638$ & $1057 / 1093$ \\
Age (years, Mean \pm s.d.) & $12.2( \pm 1.0)$ & $12.6( \pm 1.0)$ \\
BMI, kg/m² (Mean \pm s.d.) & $21.3( \pm 4.3)$ & $21.2( \pm 4.3)$ \\
Asthma (No., \%) & $19(1.5)$ & $23(1.1)$ \\
Asthma and rhinitis (No., \%) & $54(4.1)$ & $67(3.1)$ \\
Rhinitis (No., \%) & $428(33.4)$ & $764(35.5)$ \\
Not asthma, not rhinitis (No., \%) & $782(61)$ & $1296(60.3)$ \\
Allergic sensitization (No., \%) & $549(42.8)$ & $839(39.2)$ \\
Impaired lung function (No., \%) & $53(4.1)$ & $90(4.3)$ \\
$\mathrm{HCl}>1$ (No., \%) & $406(31.6)$ & $697(32.7)$ \\
\hline
\end{tabular}

$\mathrm{HCl}$, household crowding index; BMI, body mass index.

Unless otherwise indicated, data are presented as mean \pm standard deviation (s.d.).

Differences were evaluated by ANOvA or chi-square test, as appropriate.

No significant differences were found between the two groups.
Table 2 Prevalence of general and clinical variables in the sample studied, separately for gender

\begin{tabular}{lcclc}
\hline & $\begin{array}{l}\text { Prevalence } \\
(\%)\end{array}$ & $\begin{array}{l}\text { Males } \\
(\%)\end{array}$ & $\begin{array}{l}\text { Females } \\
(\%)\end{array}$ & p-value \\
\hline Asthma & 1.5 & 60 & 40 & NS \\
Rhinitis & 33.4 & 49 & 51 & NS \\
Asthma and rhinitis & 4.1 & 58 & 42 & NS \\
Not asthma, not rhinitis & 61 & 51 & 49 & NS \\
Allergic sensitization & 42.8 & 57 & 43 & $<0.001$ \\
Impaired lung function & 4.1 & 42 & 58 & NS \\
BMI $\leq$ 25 kg/m ${ }^{2}$ & 81.8 & 49 & 51 & NS \\
HCl $>1$ & 31.6 & 47 & 53 & NS \\
Depressive mood & 13.6 & 54 & 46 & NS \\
Anxious mood & 16.0 & 39 & 61 & $<0.001$ \\
Both depressive & 7.7 & 46 & 54 & NS \\
mood and anxious & & & & \\
mood & & & & \\
\hline
\end{tabular}

BMI, body mass index; $\mathrm{HCl}$, household crowding index.

Data are presented as prevalence in the overall sample and

separately for gender. Differences were evaluated by chi-square test; NS indicates not significant differences.

$(29.7 \% \rightarrow 37.9 \%)$; conversely, the likelihood of being asthmatic increased about fivefold $(0.5 \% \rightarrow 2.7 \%)$. As expected, the likelihood of being neither asthmatic nor rhinitic decreased if allergic sensitization was absent.

The Markov blanket of anxiety was composed by gender and depression. The effect of depressive mood on anxiety, measured by the relative difference, was higher among girls: for females, the difference was $52.3 \%$, whereas for males it was $42.9 \%$. A stronger effect of gender on anxiety was found among depressed subjects. The relative prevalence of anxiety was higher (by about one-third) in girls with depressive mood than in boys with depressive mood.

Table 3 shows the marginal effect of gender and depressive mood on anxious mood. The relative difference between subjects with both depression and anxiety and those with only anxiety was $47 \%$, while for females vs. males, it was $7.5 \%$. Thus, anxiety appears to have a stronger association with depression than with gender.

The Markov blanket of depressive mood was composed of anxious mood, rhinitis, and HCI. The likelihood of being depressed doubled for all conditions moving from $\mathrm{HCI} \leq 1$ to HCI $>1$, except when both anxiety and rhinitis - stronger determinants of depression than HCI - were present (Table 4).

A causal role of rhinitis on depression was found: the likelihood of being depressed increased from 11.2 to $17.7 \%$ if rhinitis was present.

\section{Discussion}

In the present study, performed on a random sample of 1283 subjects, aged 10-13, we found that rhinitis has causal role on depressive mood. Moreover, anxious mood and low socioeconomic status can contribute to induce depressive mood. Asthma is linked to depressive mood through rhinitis. A 


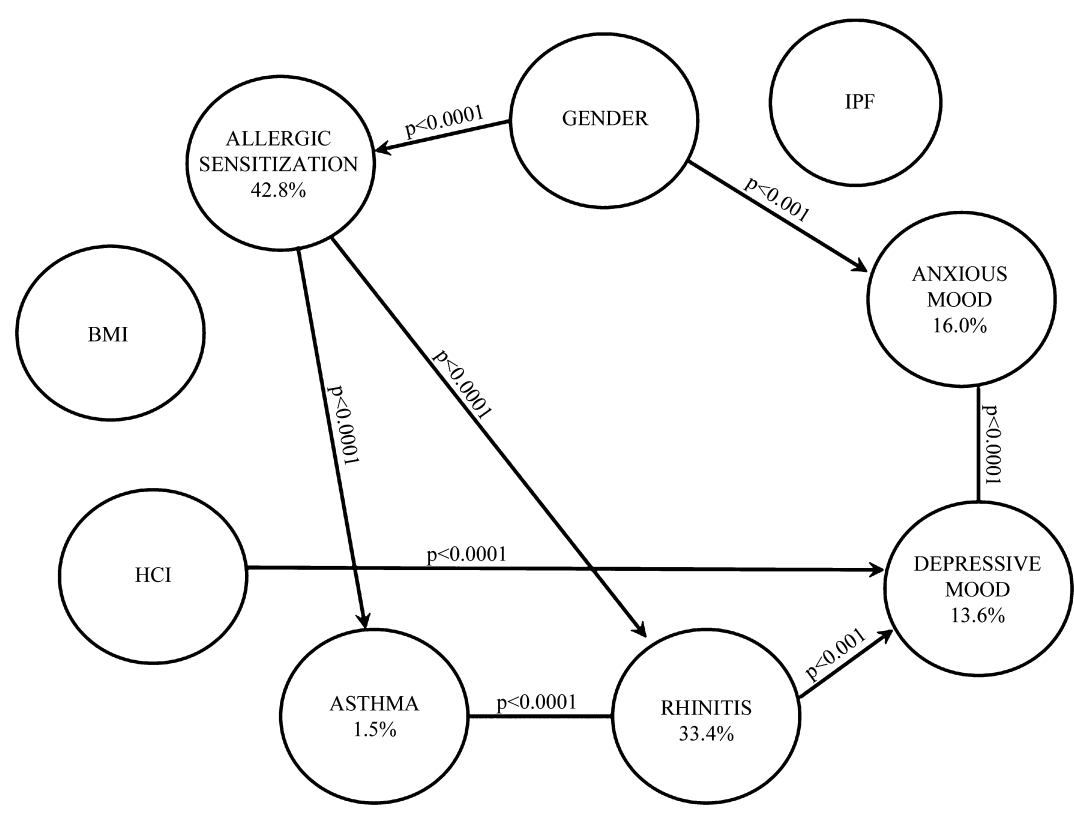

Figure 1 P-DAG summarizes the possible relationships among variables. Causal effect, between variables, is represented by arrows. The association is represented by segments. The significance of the link is indicated as p-value. The prevalence for each variables is shown.

Table 3 Marginal effect of gender (Panel A) and depression (Panel B) on anxiety

\begin{tabular}{llll}
\hline & \multicolumn{2}{c}{ Anxiety } & \\
\cline { 2 - 3 } & No & Yes & p-value \\
\hline Panel (A) Gender & & & \\
F & 80.3 & 19.7 & $<0.001$ \\
M & 87.8 & 12.2 & $<0.001$ \\
Panel (B) Depression & 90.4 & 9.6 & $<0.001$ \\
No & 43.4 & 56.6 & $<0.001$ \\
Yes & 43.4 & \\
\hline
\end{tabular}

Data are presented as prevalence of anxious mood for gender and depression, separately. The marginal effect of gender and depression on anxiety was significant $\left(\chi^{2}\right)$.

strong effect of female gender on anxiety was found, and this effect was stronger in girls with depressive mood. No effect of impaired lung function or being overweight-obese was found on either anxious or depressive mood in our sample.

In our sample, we found a higher prevalence of depressive $(13.6 \%)$ and anxious $(16 \%)$ conditions than that found in the literature. In fact, different studies have indicated that approximately $2.5-5 \%$ of children and adolescents subjects meet criteria for an anxiety disorder at any given time (20), and approximately $2.8 \%$ of children under 12 are affected by depression, with an increased prevalence during adolescence $(5.6 \%)(21)$. This difference may be due to the specific tool used to evaluate mood conditions in this age. In fact, there is general agreement that differences in methods, instruments, and populations can explain different findings among studies (22).
Table 4 Markov blanket of depression

\begin{tabular}{llllrl}
\hline \multicolumn{2}{l}{ Depression } & & & & \\
\hline $\mathrm{HCl}$ & Anxiety & Rhinitis & No & Yes & p-value \\
\hline$\leq 1$ & No & No & 95.1 & 4.9 & $<0.001$ \\
$\leq 1$ & No & Yes & 94.2 & 5.8 & $<0.001$ \\
$\leq 1$ & Yes & No & 70.1 & 29.9 & $<0.001$ \\
$\leq 1$ & Yes & Yes & 40.7 & 59.3 & $<0.001$ \\
$>1$ & No & No & 89.7 & 10.3 & $<0.001$ \\
$>1$ & No & Yes & 87.1 & 12.9 & $<0.001$ \\
$>1$ & Yes & No & 41.9 & 58.1 & $<0.001$ \\
$>1$ & Yes & Yes & 47.2 & 52.8 & $<0.001$ \\
\hline
\end{tabular}

$\mathrm{HCl}$, household crowding index.

Data are presented as prevalence (\%) of depressive mood for each combination of $\mathrm{HCl}$, Anxiety and rhinitis, showing the combined effect of these variables on depression. The marginal effect of all variables was significant $\left(\chi^{2}\right)$.

Recently, there has been a growing interest in the better definition of the psychologic burden of chronic diseases in terms of anxiety and depression symptoms (3), mostly in children affected by atopic disorders (1). Previous studies have shown that rhinitic symptoms have a significant impact on social life and school activities, influencing the patient's disease perception and psychologic characteristics (23) and mood state (24). In agreement, we found a strong association between rhinitis and depressive state.

In our study, allergic sensitization was confirmed as the common cause of asthma and rhinitis, being a predisposing factor for both. This finding is in agreement with the current 
literature (25), suggesting a strong relationship between allergy and respiratory diseases.

In addition, we found a link between asthma and rhinitis, in agreement with previous studies $(1,8,25)$. This link has been reported to produce negative physical and psychologic effects. In particular, in asthmatic allergic children, rhinitis has a strong negative effect on wellness (7).

Controversies exist relevant to a direct association between asthma and psychologic comorbidities (26). In our study, the used approach suggests that the effect of asthma on depressive mood is not direct. Similarly, gender is not directly associated with depression; instead, their link is mediated through anxiety, which is directly linked to gender. The effect of female gender on depressive mood is mediated by the predisposition of girls to anxiety, in accordance with previous studies (27). Moreover, a strong association between depression and allergy has been previously reported in females (22).

In our analysis, gender is a confounding variable in the relationship between allergic sensitization and mood state. Female gender is an indirect risk factor, for both depressive state (as the relation is mediated through anxiety) and rhinitis (as the relation is mediated through allergic sensitization).

Previous studies have shown that housing environment influences occupants' health; in particular, crowding is an important factor producing health problems, although the underlying mechanisms are not clearly established (28). We found an effect of $\mathrm{HCI}$ on depression, suggesting that socioeconomic conditions have an effect on pre-adolescent psychologic states, although other factors also act on this link. In particular, in our sample, pre-adolescents were more likely to have depressive symptoms when high HCI was associated with others depression risk factors. The effect of high HCI can be reduced by protective factors, both psychologic (i.e., absence of anxiety) and/or physical (i.e., absence of rhinitis). Thus, the assessment of atopic patients' socioeconomic status is an important step in the global approach to patients. No effect of BMI was found on mood state, in accordance with a previous study (29).

Our study presents some limitations. First, psychologic data were self-reported, so they may be biased by the difficulty that pre-adolescents have in expressing emotional states (30). Moreover, we did not explore any other psychologic or environmental variables that could influence the relation between rhinitis and mood. Longitudinal data on different personality and biologic factors could help to delve deeper into the issue.

However, it is notable that this innovative approach to analyzing the dynamic relationships between respiratory allergic conditions and mood allows us to gain further insight into the approach to allergic rhinitis in young people, stressing the role of variables regardless of their frequency.

We suggest that exploring, the psychologic dimension of rhinitic pre-adolescents subjects is a critical element of highquality clinical care performed in partnership with different specialists to increase patients' global health: the complete management of rhinitis may increase psychologic wellness and reduce disease burden. Moreover, evaluating the effect of rhinitis severity on mood state in young people may improve knowledge in this field.

\section{Acknowledgments}

We are grateful to Dr. Richard Burket for linguistic assistance with the manuscript. We are grateful to all students and their parents who participated in this study.

\section{References}

1. Bousquet J, Schünemann HJ, Samolinski B, et al. Allergic Rhinitis and its Impact on Asthma (ARIA): achievements in 10 years and future needs. $J$ Allergy Clin Immunol 2012: 130: 1049-62.

2. Slattery MJ, Essex MJ. Specificity in the association of anxiety, depression, and atopic disorders in a community sample of adolescents. $J$ Psychiatr Res 2011: 45: 788-95.

3. Waters E, Davis E, Nicolas C, Wake M, Lo SK. The impact of childhood conditions and concurrent morbidities on child health and well-being. Child Care Health Dev 2008: 34: 418-29.

4. Asher MI, Montefort S, Björkstén B, et al. Worldwide time trends in the prevalence of symptoms of asthma, allergic rhinoconjunctivitis, and eczema in childhood: ISAAC Phases One and Three repeat multicountry cross-sectional surveys. Lancet 2006: 368: 733-43

5. Galassi C, De Sario M, Biggeri A, et al. Changes in prevalence of asthma and allergies among children and adolescents in Italy: 1994-2002. Pediatrics 2006: 117: 34-42.

6. Batlles-Garrido J, Torres-Borrego J, RubíRuiz T, et al. Prevalence and factors linked to allergic rhinitis in 10 and 11-year-old children in Almería. Isaac Phase II, Spain. Allergol Immunopathol 2010: 38: 135-41.

7. Katon W, Lozano P, Russo J, McCauley E, Richardson L, Bush T. The prevalence of DSM-IV anxiety and depressive disorders in youth with asthma compared with controls. $J$ Adolesc Health 2007: 41: 455-63.

8. ISAAC Steering Committee. Worldwide variation in prevalence of symptoms of asthma, allergic rhinoconjunctivitis, and atopic eczema: ISAAC. Lancet 1998: 351: 1225-32.

9. Melki IS, Beydoun HA, Khogali M, Tamin $\mathrm{H}$, Yunis K. Household crowding index: a correlate of socioeconomic status and interpregnancy spacing in an urban setting. $J$ Epidemiol Community Health 2004: 58: 47680.
10. Newcomer P, Barenbaum E, Bryant BR TAD. Test dell'ansia e depressione dell'infanzia e nell'adolescenza. Trento: Centro Studi Erickson, 1995.

11. Asher MI, Keil U, Anderson HL, et al. International study of asthma and allergies in childhood (ISAAC): rationale and methods. Eur Respir J 1995: 8: 483-91.

12. Dreborg S, Frew A. Allergen standardization and skin tests. EAACI Position paper. Allergy 1993: 48: 48-82.

13. Miller MR, Hankinson J, Brusasco V, et al. Standardisation of spirometry. Eur Respir $J$ 2005: 26: 319-38.

14. Pistelli F, Bottai M, Carrozzi L, et al Reference equations for spirometry from a general population sample in central Italy. Respir Med 2007: 101: 814-25.

15. Gilliland FD, Berhane K, Islam T, et al Obesity and the risk of newly diagnosed asthma in school-age children. Am J Epidemiol 2003: 158: 406-15. 
16. Koller D, Friedman N. Probabilistic Graphical Models. Principles and Techniques. Cambridge, MA: The MIT Press, 2009.

17. Pearl J. Causality: Models, Reasoning, and Inference, 2nd ed. New York: Cambridge University Press, 2009.

18. Bromberg F, Margaritis D, Honavar V. Efficient Markov network structure discovery using independence tests. $J$ Artif Intell Res 2009: 35: 449-84.

19. Scutari M. Learning Bayesian networks with the bnlearn package. J Stat Softw 2010: 35: 1-22.

20. Sawyer MG, Pfeiffer S, Spence SH, et al. School-based prevention of depression: a randomised controlled study of the beyondblue schools research initiative. $J$ Child Psychol Psychiatry 2010: 51: 199-209.

21. Costello EJ, Erkanli E, Angold A. Is there an epidemic of child or adolescent depression? J Child Psychol Psychiatry 2006: 47: 1263-71.

22. Harris LR, Brown GTL. Mixing interview and questionnaire methods: Practical problems in aligning data. Practical Assessment Research \& Evaluation 2010: 15 Available from http://pareonline.net/pdf/ v15n1.pdf2010.

23. Meltzer EO. Quality of life in adults and children with allergic rhinitis. J Allergy Clin Immunol 2001: 108: 45-53.

24. Timonen M, Jokelainen J, Hakko H, et al. Atopy and depression: results from the Northern Finland 1966 Birth Cohort Study. Mol Psychiatry 2003: 8: 738-44.

25. Cibella F, Cuttitta G, La Grutta S, et al. Proportional Venn diagram and determinants of allergic respiratory diseases in Italian adolescents. Pediatr Allergy Immunol 2011: 22: 60-8.
26. Di Marco F, Santus P, Centanni S. Anxiety and depression in asthma. Curr Opin Pulm Med 2011: 17: 39-44.

27. Kirkcaldy B, Siefen G, Furnham A. Gender, anxiety-depressivity and self-image among adolescents. Eur Psychiatry 2003: 18: 50-8.

28. Fuller-Thomson E, Hulchanski JD, Hwang S. The housing/health relationship: what do we know?. Rev Environ Health 2000: 15: 109-33.

29. Benson LP, Williams RJ, Novick MB. Pediatric obesity and depression: a crosssectional analysis of absolute BMI as it relates to children's depression index scores in obese 7- to 17-year-old children. Clin Pediatr (Phila) 2013: 52: 24-9.

30. Baiardini I, Braido F, Ferraioli G, et al. Pitfalls in respiratory allergy management: alexithymia and its impact on patientreported outcomes. J Asthma 2011:48: 25-32.

\section{Supporting Information}

Additional Supporting Information may be found in the online version of this article:

Appendix S1. Bayesian networks. 